\title{
Manejo comportamental de crianças com deficiência: avaliação dos efeitos de um programa utilizando os princípios da Análise do Comportamento
}

Behavioral management of children with disabilities: evaluation of the effects of a program using the principles of Behavior Analysis

Manejo conductual de niños con discapacidad: evaluatión de los efectos de un programa utilizando los principios del Análisis del Comportamiento

\section{Thais Yazawa}

Professora doutora da Universidade de Marilia, Marília, SP, Brasil

E-mail: tatayazawa@gmail.com ORCID: https://orcid.org/0000-0001-8229-0459

Olga Maria Piazentin Rolim Rodrigues

Professora doutora da Universidade Estadual Paulista Júlio de Mesquita Filho, São Paulo, SP, Brasil

E-mail: olgarolim29@gmail.com ORCID: https://orcid.org/0000-0002-5332-256X

Recebido em 30 de agosto de 2020

Aprovado em 12 de julho de 2021

Publicado em 31 de agosto de 2021

\section{RESUMO}

Para intervir em comportamentos atípicos é necessário compreender as variáveis que os mantém. Para isso, o profissional deve compreender as variáveis das quais o comportamento é função, antes de iniciar qualquer procedimento de modificação comportamental. Os procedimentos mais utilizados são: Reforçamento Diferencial de Comportamento Alternativo (DRA) e Reforçamento Diferencial de Comportamento Incompatível (DRI). Eles possibilitam reforçar um comportamento equivalente funcionalmente, diminuindo a frequência do comportamento atípico. Os reforçamentos diferenciais, quando bem aplicados, podem trazer benefícios como o aumento do repertório de comportamentos adequados e habilidades sociais. Esse estudo pretendeu avaliar os efeitos de um programa, baseado em princípios da Análise do Comportamento, para profissionais da Saúde e Educação, sobre manejo de comportamentos atípicos em atendimento individual. O programa foi aplicado em 18 participantes das áreas da Saúde e da Educação que receberam o material autoinstrucional e foram avaliados antes e após o programa de ensino por meio de um protocolo geral. As médias apresentaram diferenças significativas, com média maior na segunda avaliação. O programa possibilitou uma formação relativamente rápida dentro das possibilidades de cada participante, sendo eficiente tanto para profissionais da Saúde como da Educação.

Palavras-chave: Análise do comportamento; Programa de ensino; Comportamentos atípicos. 
http://dx.doi.org/10.5902/1984686X54536

\section{ABSTRACT}

To intervene in atypical behaviors is necessary to understand the variables that maintain them. For this, the professional must understand the variables of which behavior is a function, before starting any behavioral modification procedure. The procedures most commonly used are: Differential Reinforcement of Alternative Behavior (DRA) and differential reinforcement incompatible behavior (DRI). They make it possible to reinforce functionally equivalent behavior, decreasing the frequency of atypical behavior. The differential reinforcements, when properly applied, can bring benefits such as increasing the repertoire of appropriate behaviors and social skills. This study aimed to evaluate the effects of a program, based on Behavior Analysis principles, for Health and Education professionals, on handling atypical behaviors in individual care. The program was applied to 18 participants from the areas of Health and Education who received the self-instructional material and were evaluated before and after the teaching program through a general protocol. The means showed significant differences, with a higher average in the second evaluation. The program enabled a relatively quick training within the possibilities of each participant, being efficient for both Health and Education professionals.

Keywords: Behavior analysis; Teaching program; Atypical behaviors.

\section{RESUMEN}

Para intervenir en comportamientos atípicos es necesario comprender las variables que los mantienen. Para ello, el profesional debe entender las variables de las cuales la conducta es una función, antes de iniciar cualquier procedimiento de modificación de la conducta. Los procedimientos más utilizados son: Refuerzo Diferencial de Conducta Alternativa (DRA) y Refuerzo Diferencial de Conducta Incompatible (DRI). Permiten reforzar comportamientos funcionalmente equivalentes, disminuyendo la frecuencia de comportamientos atípicos. Los refuerzos diferenciales, cuando se aplican correctamente, pueden traer beneficios como un aumento en el repertorio de conductas apropiadas y habilidades sociales. Este estudio tuvo como objetivo evaluar los efectos de un programa, basado en principios de Análisis de Conducta, para profesionales de la Salud y la Educación, sobre el manejo de conductas atípicas en el cuidado individual. El programa se aplicó a 18 participantes de las áreas de Salud y Educación que recibieron el material de auto-instrucción y fueron evaluados antes y después del programa de enseñanza a través de un protocolo general. Las medias mostraron diferencias significativas, con un promedio superior en la segunda evaluación. El programa permitió una formación relativamente rápida dentro de las posibilidades de cada participante, siendo eficiente tanto para los profesionales de la Salud como de la Educación.

Palabras clave: Análisis de comportamiento; Programa de enseñanza; Comportamientos atípicos. 
http://dx.doi.org/10.5902/1984686X54536

\section{Introdução}

A Análise do Comportamento (AC) é uma abordagem teórica em Psicologia, resultado da interface do Behaviorismo Radical (pressupostos teóricos, filosóficos e históricos), da Análise Experimental do Comportamento (cujos dados empíricos provêm dos métodos de investigação científica) e da Análise do Comportamento Aplicada que implica na criação e implementação de procedimentos de intervenção em Psicologia (TOURINHO, 1999; CARVALHO NETO, 2002) ou em outras áreas do conhecimento como Esporte (SOUZA; GONGORA, 2016), Saúde (MARIN; FALEIROS; MORAES, 2020) e Educação (HENKLAIN; CARMO, 2013).

A aplicação de procedimentos e princípios da Análise do Comportamento na Educação é extensa e bem documentada (KUBO; BOTOMÉ, 2001; LUNA, 2001; FRITZ; IWATA; ROLIDER; CAMP; NEIDERT, 2012; HENKLAIN; CARMO, 2013; QUERIM; IWATA; ROSCOE; SCHLICHENMEYER; ORTEGA; HURL, 2013;) com pais; (BOLSONI-SILVA; MARTURANO, 2002; PINHEIRO; HAASE; DEL PRETTE; AMARANTE; DEL PRETTE, 2006; BOLSONI-SILVA; MARIANO, 2014) e com professores (OLIVER; PRATT; NORMAND, 2015, HIGGINS; LUCZYNSKI; CARROLL; FISHER; MUDFORD, 2017). Os mesmos princípios são aplicados, também, na área da saúde (STUMPHAUZER; DAVIS, 1983; LUISELLI; BASS; WHITCOMB, 2010, MAGLIONE; KADIYALA; KRESS; HASTING; O’HANLON, 2017).

Entre os princípios da AC estão aqueles que explicam a manutenção ou não dos comportamentos de um indivíduo. A consequência produzida por uma resposta pode ter, basicamente, dois efeitos sobre ela: (a) fortalecimento ou (b) enfraquecimento (HENKLAIN; CARMO, 2013). O efeito de fortalecimento significa que uma determinada resposta tem a sua probabilidade futura de ocorrência aumentada e, as consequências que fortalecem uma resposta são chamadas de reforçadoras. Por outro lado, o efeito de enfraquecimento significa que a resposta terá menor probabilidade de ocorrer novamente no futuro e, as consequências que a enfraquecem podem ser denominadas de punitivas (MILTENBERGER; 2018).

Estudos têm utilizados os princípios da AC para o trabalho com pessoas com deficiência intelectual e com Transtorno do Espectro Autista (TEA), como, por exemplo, a Análise do Comportamento Aplicada (Applied Behavior Analysis - ABA) (NASCIMENTO; SOUZA, 2018). Um dos objetivos é promover a autonomia dessas pessoas ampliando 0 seu repertório comportamental, com ênfase nos comportamentos sociais adequados, por 
http://dx.doi.org/10.5902/1984686X54536

meio de observações sistemáticas e registro sistemático dos comportamentos. Entre os pontos fortes desse programa está a participação ativa dos pais.

Heitzman-Powell, Buzhardt, Rusinko e Miller (2014) avaliaram um treino para pais de crianças com TEA que moram em áreas remotas, baseado em ABA. O treino para pais pautou-se na grande quantidade de crianças necessitando de intervenção e, ao envolvêlos, aumentariam as oportunidades de aprendizado, promoveriam generalizações e reduziriam os valores dos tratamentos. O programa de treinamento OASIS, utilizando as técnicas de intervenção validadas empiricamente via ABA, foi adaptado para os pais em uma linguagem mais acessível e modificado para plataformas de videoconferência. $O$ conteúdo foi dividido em oito módulos e, enquanto critérios, o participante deveria acertar 90\% das avaliações para avançar. Sete pais participaram do estudo, realizando as atividades e os treinamentos online. Além das aulas, os pais receberam brinquedos préescolares e materiais educacionais. Os treinadores ofereceram instruções e feedback após as discussões sobre os módulos estudados. Dos 48 itens aplicados no pré e pós-testes com os pais, os índices de acertos aumentaram de 53,13\% para 92,25\%.

Outro programa para pais, baseado em evidências com base em AC, o "Strongest Families" foi desenvolvido por Olthuis et al. (2018). O programa era composto por material escrito, vídeos e sessões de treinamento por telefone, com foco na redução de problemas comportamentais. Participaram 172 cuidadores primários de crianças de seis a doze anos. Foram realizadas medidas de problemas de comportamento, das práticas parentais, do estresse parental antes e depois da participação no programa. A análise dos dados demonstrou que houve significante redução nos problemas comportamentais, com melhorias mais significativas após a 10ª semana de intervenção. Depois da intervenção também diminuiu a disciplina inconsistente e houve, também, significativa diminuição do estresse parental.

Bagaiolo et al. (2017) realizaram um treino de pais baseado em Análise do Comportamento, durante 22 semanas, utilizando vídeo-modelação. Participaram 67 pais que foram randomizados em dois grupos: 34 em intervenção e 33 no grupo controle. Catorze vídeos foram filmados para o manejo de comportamentos disruptivos, "Prompting hierarchy", que é um sistema de dicas que permite à criança oportunidades para se comunicar, indo do mais ao menos diretivo, avaliação de preferências, aquisição de melhor contato visual e melhorar a atenção e obediência. Os pais foram ensinados a utilizar a 
http://dx.doi.org/10.5902/1984686X54536

estratégia de tentativas discretas (DTT). Após a intervenção, foram observadas melhoras significativas na dimensão Obediência (70,6\%).

Preparar os pais para lidar com suas crianças com TEA ou deficiência intelectual que apresentam é tão importante quanto capacitar profissionais que trabalham diretamente com essa população em atendimento ambulatorial, como os profissionais da Saúde ou, diariamente e em tempo maior, como os profissionais da Educação. Luiselli, Bass e Whitcomb (2010) realizaram um estudo para avaliar um programa sistemático de treinamento para ensinar princípios da Análise do Comportamento Aplicada a 35 funcionários recémcontratados em um serviço de residência para adultos com deficiência intelectual. Três aspectos do novo trabalho foram contemplados: avaliação, suporte comportamental e aquisição de habilidades. Foram utilizados instruções didáticas, exercícios práticos e demonstrações em vídeo. O desempenho foi medido por avaliações pré e pós-testes. No préteste o total de acertos foi de 76,8\% e 92\% no pós-teste no Módulo 1 de Avaliação. No Módulo 2, de Suporte Comportamental, o total de acertos $75 \%$ e, no pós-teste foi de $89,2 \%$. As pontuações do Módulo 3 de aquisição de habilidades foram de 62,4\% no pré-teste e 90,4\% no pós-teste. É, então, possível que os conhecimentos produzidos pela Psicologia e, em especial, pela Análise do Comportamento, sejam ensinados para outros profissionais que trabalham com pessoas com deficiência intelectual e/ou TEA.

Quatro díades professor de Educação Especial e seu aluno foram alvo de intervenção em um estudo conduzido por Reed et al. (2007) com o objetivo de diminuir problemas de comportamento em sala de aula. Os problemas de comportamento de três das díades diminuíram significativamente após a intervenção.

Procedimentos com reforçamento diferencial têm sido utilizados em intervenções realizadas diretamente com pessoas com TEA ou deficiência intelectual severa, com pais e, também, com profissionais que prestam atendimento a elas em instituições educacionais ou de saúde. Vollmer e Iwata (1992) descreveram os reforçamentos diferenciais com estratégias metodológicas para o tratamento de desordens comportamentais, incluindo comportamentos disruptivos, autolesivos, agressivos ou outros.

"Os procedimentos baseados em reforçamentos diferenciais são associados a diversos benefícios. Sejam implementados como um tratamento simples ou em conjunto com outros procedimentos (...) reforçamentos diferenciais não requerem que o indivíduo seja removido do ambiente educativo (como nos procedimentos de time out) ou não requerem a aplicação de uma consequência aversiva contingente (como nos procedimentos de punição)" (p. 394). 
http://dx.doi.org/10.5902/1984686X54536

São reforçamentos diferenciais quando as consequências reforçadoras estão disponíveis após a emissão de comportamentos adequados, sendo estes quaisquer outros comportamentos (Differential reinforcement of Other behavior - DRO), após a emissão de comportamentos incompatíveis com os comportamentos que deverão diminuir de frequência (Differential reinforcement of Incompatible behavior - DRI) e, também, após a emissão de quaisquer outros comportamentos alternativos que não o comportamento-alvo (Differential reinforcement of alternative behavior - DRA) (STEINBRENNER; HUME; ODOM et al., 2020).

Procedimentos que utilizam reforçamentos diferenciais, quando bem aplicados, podem trazer benefícios como o aumento do repertório adequado de habilidades sociais e de comunicação de pessoas com deficiência intelectual e autismo. A Análise do Comportamento (AC), enquanto ciência, tem demonstrado potencial tecnológico no desenvolvimento de repertório social adequado junto a pessoas com diferentes expressões de deficiência. Parece interessante viabilizar o acesso ao conhecimento de seus princípios a outros profissionais não psicólogos (FORNAZARI, 2005), como pais, professores e outros profissionais da saúde que prestam serviços para esta população ou que convivem diariamente com ela.

Um professor foi treinado a utilizar o DRA com fichas, para reduzir comportamentos problemas (ecolalias, choro, bater em objetos, agressão e vocalizações inadequadas) de três crianças de cinco a sete anos, em um estudo conduzido por Mueller et al. (2003). As fichas eram trocadas por 15 segundos de acesso a itens preferidos após a emissão de comportamentos adequados (obediência e adesão à tarefa). Ao dar as fichas, o professor lembrava a criança sobre a relação entre o comportamento adequado emitido, o número de fichas e o tempo de acesso. Os comportamentos de obediência e aceitabilidade das atividades aumentaram nas fases de DRA, DNRA (reforçamento negativo diferencial de comportamento alternativo) e NCR (reforçamento não contingente). O DRA e o NCR apresentaram melhores resultados que o DRNA, apresentando níveis mais baixos do comportamento atípicos na pós-intervenção do que na linha de base.

A Análise Funcional (AF) é uma ferramenta útil tanto para a avaliação como, posteriormente, na intervenção, auxiliando na identificação da função do comportamento e quais as consequências que o mantém e que podem, posteriormente, serem modificadas (MATOS, 1999). Ensinar pais e profissionais a realizarem a análise funcional torna-os capazes de implementar mudanças no ambiente, alterando o oferecimento das consequências que mantém comportamentos indesejáveis, lançando mão de reforçamento diferencial, por exemplo. Apesar do treino de pais/profissionais dispender de tempo e energia, 
http://dx.doi.org/10.5902/1984686X54536

eles ocasionam um impacto positivo na família, pois possibilita que a intervenção seja feita no ambiente natural (BOOTH; GALLAGHER; KEENAN, 2018), aumentando as possibilidades de intervenção para fora da clínica. Além da AF, ainda há outra estratégia prática para determinar a função do comportamento, como a Avaliação Funcional Descritiva, que pode ser realizada por meio de escalas, entrevistas com pais e professores e observação direta (BRUHN; BALINT-LAGEL; TROUGHTON et al, 2015). Tanto os dados da AF, quanto da Avaliação funcional descritiva (FBA) possibilitam auxiliar na definição de quais reforçadores e quais tipo de reforçadores devem ser utilizados ao se implementar uma intervenção

Utilizando videoconferência, Higgins et al. (2017) ensinaram profissionais não psicólogos a realizar análise funcional de comportamentos-problema que ocorriam nas sessões de atendimento. A partir do início do curso, em todas as sessões foram emitidas taxas menores de problemas de comportamento, quando comparados com a linha de base, tanto para o profissional recém-capacitado, quanto para o profissional mais experiente. $O$ procedimento mostrou-se viável economicamente, possibilitando que profissionais capacitados treinem outros profissionais iniciantes.

Os estudos apontaram que, tanto os professores, quanto os profissionais de saúde e demais prestadores de serviços para as pessoas com deficiência intelectual e suas famílias podem ser capacitados para uma melhor aplicação de suas habilidades profissionais específicas, quando associadas a procedimentos de manejo do comportamento social de seus pacientes. O desenvolvimento das habilidades de manejo de comportamento próadesão aos procedimentos específicos da área podem ser ensinados a todos os profissionais não psicólogos.

Para construir um programa de ensino "é necessário caracterizar as necessidades sociais da população e possibilidades de atuação derivadas dessas necessidades" (KIENEN; KUBO; BOTOMÉ, 2013, p. 482). Neste sentido, ensinar aos profissionais o manejo de comportamentos inadequados de seus clientes ou alunos aumenta a probabilidade de sucesso na aprendizagem de repertórios comportamentais diretamente relacionados às suas necessidades de desenvolvimento.

O manejo de comportamentos pode ser ensinado para professores e profissionais da saúde que atendem pessoas que apresentam graves problemas de comportamento, para que eles possam ser instrumentalizados de maneira adequada. Utilizando os princípios da Análise do Comportamento para o desenvolvimento de comportamentos adequados, 
http://dx.doi.org/10.5902/1984686X54536

materiais foram elaborados para ensino de pais (HAMADA; FORNAZARI, 2014) e para profissionais da saúde (YAZAWA; FORNAZARI, 2015). Comportamentos-objetivos foram decompostos e o conteúdo foi elaborado em pequenos passos, com dificuldade crescente e apresentando feedback imediato dos conteúdos estudados.

Hamada (2014) desenvolveu um material didático para pais, com o objetivo de auxiliálos a diminuir a ocorrência dos comportamentos atípicos em crianças com deficiência intelectual. O material foi aplicado em sete familiares de pessoas que apresentavam comportamentos atípicos. A autora considerou o material validado socialmente, pois $42 \%$ dos participantes afirmaram que aprenderam alguma coisa após o contato com o material.

Além da escola, o sistema de saúde também presta serviços para as pessoas e para as famílias das pessoas com deficiência. Desta forma, ele precisa estar preparado para atender a população e lidar com comportamentos que podem atrapalhar seu envolvimento no atendimento especializado. Yazawa (2015) utilizou o material de Hamada (2014) adaptando-o para uso com profissionais da saúde. Participaram 11 profissionais, entre eles, fisioterapeutas, terapeutas ocupacionais, fonoaudiólogos e psicólogos. Os participantes receberam um material impresso, contendo três módulos, sobre os princípios básicos da Análise do Comportamento, com exemplos específicos do seu cotidiano. Após a leitura do texto didático, o participante realizava exercícios relacionados aos conteúdos, obtendo feedback imediato, presente no caderno. Cada participante era responsável por seu próprio ritmo de aprendizagem, avançando no material de acordo com seu desempenho e tempo de dedicação ao mesmo. Os resultados observados ao final de cada módulo foram de 91,75\% de acertos no Módulo 1, 80,9\% no Módulo 2 e 91,89\% de acertos no Módulo 3. Os dados obtidos na Unidade II apontaram para a necessidade de mudanças, concretizadas no presente estudo. Os resultados no pós-teste foram significativamente superiores aos do préteste. Enquanto lacuna deste estudo, o aprendiz foi responsável por suas correções, o que pode ter enviesado os resultados obtidos, sugerindo que, entre os módulos, haja uma avaliação externa.

Considerando a necessidade de capacitar profissionais da saúde e da educação para lidar com os comportamentos atípicos, que dificultam as atividades laborais de suas competências, a Análise do Comportamento, enquanto área do conhecimento, pode auxiliar profissionais não-psicólogos com o conhecimento científico produzido. Além da formação desses profissionais, é importante verificar, na prática, se mudanças comportamentais dos profissionais geram mudanças comportamentais em seus pacientes ou alunos. $\mathrm{O}$ uso de 
http://dx.doi.org/10.5902/1984686X54536

avaliações sistemáticas externas e filmagens de profissionais e pacientes/alunos em situações reais pode auxiliar a observação do aprendido na prática.

Dentre as diversas possibilidades de ensino e formação continuada com o objetivo de promover a aquisição de novos conhecimentos, os cursos autoinstrucionais têm sido utilizados pelas instituições de ensino (CONSTANCIO; NOGUEIRA; COSTA, 2016). Tal característica é compatível com a configuração da vida moderna, em que muitos profissionais dispõem de pouco tempo para se aperfeiçoar ou atualizar-se. As capacitações autoinstrucionais, sobretudo as informatizadas, com o advento do EAD, estão se popularizando no Brasil (DE BONA, 2010; FAÉ, 2012; CONSTANCIO; NOGUEIRA; COSTA, 2016; BRASIL, 2016; YOSHIY; KIENEN, 2019).

Considerando a necessidade dos profissionais da Saúde e da Educação em adquirir os conhecimentos produzidos pela $\mathrm{AC}$, que aperfeiçoariam sua atuação no manejo comportamental e a lacuna na literatura brasileira de estudos que avaliem a pertinência desta formação, o presente estudo pretendeu responder às questões: o desempenho de profissionais da Saúde e de Educação sobre Análise do Comportamento e sua aplicação ao manejo de comportamentos atípicos melhorariam após participarem de um programa de ensino? Haveria diferença entre o desempenho dos profissionais da Saúde e da Educação? Haveria, também, diferenças no desempenho de profissionais que conhecessem anteriormente AC?

Pretendeu-se, neste estudo, avaliar os efeitos de um programa, baseado em princípios da Análise do Comportamento, para ensino de manejo de comportamentos atípicos em atendimento individual, para profissionais da Saúde e Educação. Os objetivos específicos foram: descrever e comparar o desempenho das participantes no pré e pós-teste da avaliação geral; considerando professores e profissionais da saúde e, se tinham ou não, conhecimento prévio em Análise do Comportamento.

\section{Método}

\section{Participantes}

A pesquisadora entrou em contato com instituições das cidades de Tupã, Assis, Marília, Garça, Pompéia, Presidente Prudente, Duartina, Agudos, São Manuel, Botucatu, Pederneiras, Lençóis Paulista. Destas, aderiram ao estudo as instituições das cidades de Tupã, Pompéia, Presidente Prudente, Agudos, São Manuel e Pederneiras. Do conjunto das 
http://dx.doi.org/10.5902/1984686X54536

cidades participaram 18 profissionais da saúde (fonoaudiólogos, fisioterapeutas, psicólogos, enfermeiros, terapeutas ocupacionais e pedagogos) alocados em seis instituições e que atendiam individualmente crianças ou adolescentes com deficiências diversas que apresentavam comportamentos atípicos. As participantes estão descritas na Tabela 1.

As profissionais participantes da pesquisa eram $100 \%$ do sexo feminino, sendo a maioria destas (50\%) com mais de 41 anos. As professoras (33,3\%) compõem a maioria das participantes da capacitação, seguidas por psicólogas (22,2\%) terapeutas ocupacionais, fisioterapeutas $(16,6 \%)$ e fonoaudiólogas $(11,1 \%)$. Quanto à formação complementar, 83,3\% delas realizaram especializações e 38,8\% cursos de curta duração. Com relação ao tempo de atuação na área, 50\% tem mais de seis anos de experiência na área. Delas 38,8\% afirmaram não conhecerem a Análise do Comportamento.

Tabela 1 - Dados sociodemográficos dos participantes

\begin{tabular}{ccc}
\hline Aspectos & N & $\%$ \\
\hline Sexo & 0 & 0 \\
Masculino & 18 & $100 \%$ \\
Feminino & & \\
Idade & 4 & $22,2 \%$ \\
De 24 a 31 anos & 5 & $27,7 \%$ \\
De 32 a 40 anos & 9 & $50,1 \%$ \\
41 anos ou mais & & \\
Formação & 6 & $33,3 \%$ \\
Pedagogia & 3 & $16,6 \%$ \\
Terapia ocupacional & 3 & $16,6 \%$ \\
Fisioterapia & 2 & $11,1 \%$ \\
Fonoaudiologia & 4 & $22,2 \%$ \\
Psicologia & 15 & $83,3 \%$ \\
\hline Formação complementar & 7 & $38,8 \%$ \\
Especialização & & \\
Cursos de curta duração & 8 & $44,4 \%$ \\
\hline Tempo de atuação na área & 9 & $50 \%$ \\
O a 5 anos & 1 & $5,5 \%$ \\
6 a 15 anos & & \\
Mais de 15 anos & 10 & $55,6 \%$ \\
Conhece Análise do & 8 & $44,4 \%$ \\
Comportamento? & & \\
Sim & & \\
Não & &
\end{tabular}

Fonte: Elaborada pelas autoras (2017).

\section{Local}

A pesquisa foi realizada em seis instituições que atendem crianças com deficiência intelectual e TEA, no interior de São Paulo. 
http://dx.doi.org/10.5902/1984686X54536

\section{Instrumentos}

a. Material didático: Graves problemas de comportamento no atendimento em saúde: como lidar?

O material instrucional Graves problemas de comportamento no atendimento em saúde: como lidar? (YAZAWA; FORNAZARI; RODRIGUES, 2018), adaptado de Yazawa e Fornazari (2015), contou com três módulos, contendo textos e exercícios dissertativos e de múltipla escolha para avaliação do material lido com crivo de autocorreção. Para a confecção do material alguns princípios básicos da Programação de Ensino (BOTOMÉ. 1981; KELLER; SHERMAN, 1974) foram considerados: 1) Pequenos passos: adotando pequenas unidades de ensino para facilitar a aprendizagem e ir aumentando a dificuldade aos poucos para uma aprendizagem sem erros; 2) Resposta ativa do aprendiz; 3) Verificação imediata: refere-se ao feedback imediato sobre seu desempenho, otimizando a aprendizagem; 4) Ritmo individual: cada um aprende em seu ritmo, avançando de acordo com sua capacidade e desempenho e, 5) Teste de avaliação: que o aluno faz de cada unidade de ensino, para revisar e aperfeiçoar seu repertório sobre o assunto. O programa de ensino está detalhado no Quadro 1.

Quadro 1 - Conteúdo do material didático

\begin{tabular}{|c|c|c|c|c|}
\hline Módulo & Conteúdo geral & Temas & $\begin{array}{l}\text { Número de } \\
\text { páginas }\end{array}$ & Exercícios \\
\hline \multirow{7}{*}{1} & \multirow{7}{*}{$\begin{array}{l}\text { Princípios da } \\
\text { AC }\end{array}$} & $\begin{array}{l}\text { Antecedente, resposta e } \\
\text { consequência }\end{array}$ & 4 & 2 \\
\hline & & Contingência & 4 & 1 \\
\hline & & Punição & 4 & 6 \\
\hline & & Extinção & 3 & 5 \\
\hline & & Discriminação e generalização & 5 & 8 \\
\hline & & Reforço & 3 & 4 \\
\hline & & Revisão & 6 & 2 \\
\hline \multirow{5}{*}{2} & \multirow{5}{*}{$\begin{array}{l}\text { Análise } \\
\text { Funcional }\end{array}$} & Conceito de Análise Funcional & 3 & 3 \\
\hline & & Registro de respostas & 3 & 0 \\
\hline & & Registro dos consequentes & 3 & 0 \\
\hline & & Registro dos antecedentes & 11 & 4 \\
\hline & & $\begin{array}{l}\text { Funções do comportamento e } \\
\text { Análise funcional a partir da folha } \\
\text { de registros }\end{array}$ & 7 & 4 \\
\hline 3 & $\begin{array}{l}\text { Intervenção via } \\
\text { Esquemas de } \\
\text { Reforçamento }\end{array}$ & Reforçamentos diferenciais & 32 & 27 \\
\hline
\end{tabular}

Fonte: Elaborado pelas autoras (2018).

$\mathrm{Na}$ adaptação do material foram incluídas mais ilustrações e o Módulo 2 foi revisto, com informações mais detalhadas e mais exemplos práticos foram apresentados, devido 
http://dx.doi.org/10.5902/1984686X54536

ao número de materiais em branco que voltaram nesta parte específica no estudo de Yazawa (2015). O material foi dividido em módulos e foram incluídos protocolos de avaliação entre eles, aplicados antes e depois de cada módulo, além da avaliação geral, respondida antes e depois do programa.

b. Protocolo de avaliação intermódulos e geral para pré e pós-intervenção

O Protocolo de Avaliação Geral, elaborado para esse estudo, era composto de 10 questões objetivas, cujo enunciado trazia uma situação-problema na qual o participante deveria escolher a conduta que the parecia mais adequada, dentro do contexto clínico ou escolar, para o manejo comportamental de seus pacientes ou alunos.

Os Protocolos de avaliação intermódulos, também elaborados para esse estudo, versaram sobre os conteúdos de cada módulo (1, 2 ou 3). O Protocolo do Módulo 1 era composto de 12 questões objetivas, que versavam sobre os princípios básicos da AC, o Protocolo do Módulo 2, também composto por 12 questões objetivas, abordava o uso da Análise Funcional e, o Protocolo do Módulo 3 era composto por 10 questões objetivas sobre a aplicação do DRA e do DRI.

\section{Procedimento para a coleta dos dados}

Após aprovação do Comitê de Ética, as instituições foram contatadas para a apresentação do projeto de pesquisa e suas etapas. Posteriormente à anuência, foi realizada uma reunião com a coordenação e os profissionais. Identificados os participantes, estes preencheram o Termo de consentimento livre e esclarecido e o Protocolo geral de avaliação. Foi esclarecido que, caso o critério de aprovação (80\% de acertos) na avaliação de cada Módulo não fosse obtido, um novo prazo para a avaliação seria combinado. Se alcançado o critério de $80 \%$ de acertos, seria entregue o material do próximo Módulo. Em seguida, foi entregue o material do Módulo 1, com instruções de como preenchê-lo e acordado com cada participante um prazo médio para a realização da avaliação. O mesmo procedimento foi realizado com os demais módulos. Depois de obtido o critério no Módulo 3, as participantes responderam ao Protocolo Geral do programa.

\section{Procedimento de análise}

Análises intergrupos foram feitas comparando o desempenho das participantes antes e depois do curso. Também, comparações intragrupos foram feitas considerando o grupo de profissionais da saúde, com 12 participantes e da educação, com 06 participantes, assim 
http://dx.doi.org/10.5902/1984686X54536

como se o conhecimento destas em Análise do Comportamento poderia influenciar em seu processo de aprendizagem. Para a análise, foi utilizado o teste inferencial não paramétrico de Wilcoxon.

\section{Resultados}

Com a finalidade de avaliar o programa de ensino das profissionais, foi aplicado o Protocolo Geral antes e depois da finalização do programa. Os dados da Tabela 2 mostram que houve diferença significativa entre as duas avaliações $(p=0,000)$, com média maior na segunda avaliação.

Tabela 2 - Resultados médios dos pré e pós-teste da avaliação geral

\begin{tabular}{llllll}
\hline & Média & Mediana & DP & Mínimo & Máximo \\
\hline Pré teste & 6,88 & 7 & 1,23 & 5 & 9 \\
Pós teste & 8,88 & 9 & 1,32 & 5 & 10 \\
\hline $\boldsymbol{P}$ & $<0.001$ & & & & \\
\hline
\end{tabular}

Fonte: Elaborada pela autora (2020).

A Tabela 3 apresenta a frequência de participantes que tiveram acertos no Protocolo de avaliação geral antes e depois do programa. Observa-se que $16(88,9 \%)$ das participantes acertaram oito ou mais questões na segunda avaliação contra seis (33,3\%) na primeira avaliação.

Tabela 3 - Frequência absoluta e relativa de acertos do Protocolo na amostra geral nas duas avaliações gerais

\begin{tabular}{ccccc}
\hline Acertos & \multicolumn{2}{c}{ Avaliação pré } & \multicolumn{2}{c}{ Avaliação pós } \\
\hline & $\mathbf{N}$ & $\%$ & $\mathbf{N}$ & $\%$ \\
\hline $\mathbf{5}$ & 2 & 11,1 & 1 & 5,6 \\
$\mathbf{6}$ & 6 & 33,3 & 0 & 0 \\
$\mathbf{7}$ & 4 & 22,2 & 1 & 5,6 \\
$\mathbf{8}$ & 4 & 22,2 & 3 & 16,7 \\
$\mathbf{9}$ & 2 & 11,1 & 6 & 33,3 \\
$\mathbf{1 0}$ & 0 & 0 & 7 & 38,9 \\
\hline
\end{tabular}

Fonte: Elaborada pelas autoras (2020).

Considerando que professoras compunham mais do que $30 \%$ da amostra e realizavam atendimentos em pequenos grupos, optou-se por separá-las dos profissionais da saúde. Comparou-se, então, as avaliações gerais antes e depois do programa do Grupo 1 (professoras) e do Grupo 2 (profissionais da saúde). Observa-se que houve melhora da pontuação média da primeira para a segunda avaliação para os dois grupos. Todavia, a diferença significativa da primeira avaliação para a segunda foi encontrada no grupo de 
http://dx.doi.org/10.5902/1984686X54536

outras profissionais $(p=0,003)$, com pontuação média maior na segunda avaliação, conforme mostra a Tabela 4.

Tabela 4 - Comparação entre grupos de professores e profissionais antes e depois da participação no programa

\begin{tabular}{llllll}
\hline Grupos & Pré & & Pós & & $\boldsymbol{P}$ \\
\hline & Média & DP & Média & DP & \\
\hline Professores $\mathbf{n = 6}$ & 6.5 & 1,37 & 8,33 & 1,75 & 0,071 \\
Profissionais da saúde $\mathbf{n = 1 2}$ & 7,8 & 1,16 & 9,16 & 1,02 & 0,003 \\
\hline
\end{tabular}

Fonte: Elaborada pelas autoras (2020).

Das participantes, cerca de 56\% relataram conhecer Análise do Comportamento, apesar de não usar seus princípios nos atendimentos realizados. Comparou-se, então, o desempenho nas duas avaliações gerais considerando o conhecimento prévio ou não dos princípios de AC (Tabela 5). Houve diferença significativa tanto no grupo de profissionais com conhecimento prévio ( $p=0,007)$, quanto no grupo de profissionais sem conhecimento prévio $(0,024)$, com média maior no pós-teste para os dois grupos.

Tabela 5 - Comparação entre grupos de participantes que tinham conhecimento prévio de AC e de profissionais que tiveram contato pela primeira vez

\begin{tabular}{llllll}
\hline Grupos & Pré-teste & Pós-teste & P \\
\hline & Média & DP & Média & DP & \\
\hline Conhecimento prévio AC $(\mathbf{n = 1 0})$ & 7,2 & 1,3 & 9,2 & 1,0 & $\mathbf{0 , 0 0 7}$ \\
Sem conhecimento prévio AC $(\mathbf{n}=\mathbf{8})$ & 6,5 & 0,9 & 8,5 & 1,6 & $\mathbf{0 , 0 2 4}$
\end{tabular}

Fonte: Elaborada pelas autoras (2020).

\section{Discussão}

As adaptações realizadas no material do programa instrucional Graves problemas de comportamento: Como lidar tiveram como objetivo aumentar a probabilidade de acertos e melhor aproveitamento das participantes. Assim, houve um aumento no número de módulos e foram elaboradas avaliações entre os módulos com critério de $80 \%$ de acertos para passar de um módulo a outro. Como Botomé (1981) e Keller e Sherman (1974) preconizaram, a autoavaliação realizada no material possibilitou a sua revisão, melhorando seus pontos deficitários, possibilitando um melhor aproveitamento de cada módulo. Segundo Keller e Sherman (1974), a análise do material é tarefa do instrutor, por inadequabilidade de seu conteúdo ou estrutura. A inclusão de um protocolo geral, avaliando o desempenho dos participantes antes e depois possibilitou a análise da 
http://dx.doi.org/10.5902/1984686X54536

qualidade do curso oferecido, tanto considerando a especificidade do profissional, da Saúde ou da Educação e, ainda, o conhecimento prévio sobre AC dos participantes.

O novo formato do programa de ensino possibilitou uma formação relativamente rápida para as profissionais que atendem as pessoas com deficiência intelectual, uma importante qualidade dos cursos autoinstrucionais (CONSTANCIO; NOGUEIRA; COSTA, 2016), atendendo assim, um de seus objetivos. Considerando a amostra total das participantes observaram-se melhoras após o contato com o material desenvolvido para este estudo, com média significativamente maior na avaliação após o programa, que sinaliza que a compreensão dos conceitos melhorou. Tais resultados corroboram os encontrados por Luiselli, Baso e Whitcomb (2010) que obtiveram sucesso ao ensinar esses mesmos princípios para profissionais não psicólogos.

Os conceitos estudados têm como objetivo auxiliar os profissionais, na sala de aula ou durante os atendimentos, no manejo de comportamentos atípicos, que podem dificultar ou impedir o acesso do aluno ou cliente à aprendizagens especificas, conforme lacuna apontada por Koegel et al. (2012). O programa possibilitou que os participantes tivessem acesso a conceitos e como aplicá-los no dia a dia, como, por exemplo: como realizar uma AF e, também, como aplicar procedimentos de reforçamentos diferenciais, práticas que podem melhorar suas atividades laborais. Os dados levantados vão ao encontro do que Moore et al. (2002) sugeriram em seu estudo, que, com treino adequado, professores podem aprender a implementar AF de forma correta.

Considerando a formação dos participantes, os resultados obtidos por profissionais da saúde na segunda avaliação foram significativamente melhores do que os das professoras que também obtiveram bons resultados. Digennaro et al. (2007) ressaltaram a importância de capacitar esses profissionais por estarem na linha de frente no atendimento à criança com deficiência ou TEA. A proposta inicial do programa de intervenção se limitava aos profissionais da Saúde e, por solicitação das instituições, os professores também foram inseridos.

A média das participantes com conhecimento prévio em AC e sem conhecimento prévio em AC aumentou também, compreendemos, assim, que as participantes sem conhecimento prévio se apropriaram dos conceitos ensinados. A variação foi maior na média do pós-teste das participantes que conheciam a $A C$, chegando quase à nota máxima da avaliação. Tais resultados apontaram para a importância de retomar conceitos já conhecidos de AC, mas, também, dar oportunidade a outros profissionais de aprendê- 
los, como o estudo de Yazawa (2015). Estudo conduzido por Marin, Faleiros e Moraes (2020) mostrou que é possível ensinar profissionais da Saúde a aplicarem os conceitos de AC em seus contextos de trabalho assim como Henklain e Carmo (2013) o fizeram com profissionais da Educação.

Ensinar os princípios e conceitos da AC e verificar se os profissionais aprenderam é importante, mas não dá a dimensão se estão realmente utilizando-os na prática. Estudos como os de Mueller et al. (2003) e Higgins et al. (2017) mostraram que é possível ensinar os conceitos na modalidade de formação em serviço, onde o profissional tem a teoria associada à prática, uma aprendizagem mais eficiente e duradoura dos conceitos da AC.

\section{Considerações finais}

Este estudo teve por objetivo avaliar os efeitos de um programa de ensino, baseado em princípios da Análise do Comportamento, para profissionais da Saúde e Educação sobre manejo de comportamentos atípicos em atendimento individual ou em sala de aula. Os resultados apontaram para o aumento do repertório dos profissionais sobre o tema, tanto da Educação como da Saúde, considerando aqueles que já tinham tido acesso aos conceitos anteriormente como aqueles que estavam tendo um contato inicial com eles.

Enquanto limitações deste estudo, a aprendizagem das participantes foi medida apenas pelo desempenho do conhecimento de conceitos da AC, AF e Reforçamento Diferencial na avaliação geral antes e depois do programa. Sugere-se, para estudos futuros, que a aprendizagem dos participantes seja avaliada comparando a utilização desses conceitos na prática do profissional antes e após a participação no programa para observar se há melhoras.

A Análise do Comportamento é uma ciência que muito pode contribuir com as outras áreas de conhecimento, em uma parceria produtiva. Todavia, o pequeno número de participantes de cada área inviabilizou análises específicas com cada um deles. Os conhecimentos produzidos pela academia devem chegar até àqueles mais necessitados. E estudos como esse podem melhorar a qualidade de vida das crianças atendidas e a prática laboral dos profissionais que atendem as crianças com deficiência intelectual que apresentam comportamentos atípicos, extrapolando o conhecimento além da Psicologia e perpassando para as demais áreas da Saúde e da Educação. 
http://dx.doi.org/10.5902/1984686X54536

\section{Referências}

BAGAIOLO, Leila F.; PACÍFICO, Cláudia R.; MOYA, Ana Cláudia C.; MIZAEL, Lucimara F.; JESUS, Fiama S.; ZAVITOSKI Marina; SASAKI, Tatiana; AZEVEDO, Graccielle R. C. Capacitação parental para comunicação funcional e manejo de comportamentos disruptivos em indivíduos com Transtorno do Espectro Autista. Cadernos de Pós-Graduação em

Distúrbios do Desenvolvimento, São Paulo, v. 18, n. 2, p. 46-64, 2018. http://dx.doi.org/10.5935/cadernosdisturbios.v18n2p46-64 Acesso em mar 2021

BOLSONI-SILVA, Alessandra T.; MARIANO, Maria L. Práticas educativas de professores e comportamentos infantis, na transição ao primeiro ano do Ensino Fundamental. Estudos e Pesquisas em Psicologia, Rio de Janeiro, v. 14, n. 3, p. 814-833, 2014. Disponível em: http://pepsic.bvsalud.org/scielo.php?script=sci_arttext\&pid=S180842812014000300007\&lng=pt\&nrm=iso Acesso em 31 jul. 2020

BOLSONI-SILVA, Alessandra T.; MARTURANO, Edna M. Práticas educativas e problemas de comportamento: uma análise à luz das habilidades sociais. Estudos de Psicologia, Natal, v. 7, n. 2, p. 227-235, 2002. Disponível em:

https://www.scielo.br/pdf/epsic/v7n2/a04v07n2.pdf Acesso em 31 jul. 2020

BOOTH, Nichola; GALLAGHER, Stephen; KEENAN, Mickey. Autism, interventions and parent training. Psicologija praktikai, v. 57, p. 74-94, 2018. Disponível em: https://pureadmin.qub.ac.uk/ws/portalfiles/portal/164750344/11911_Article_Text_14431_1_ 10_20181015.pdf Acesso em 09 de abr. 2020.

BOTOMÉ, Sílvio P. Objetivos comportamentais no ensino: A contribuição da Análise Experimental do Comportamento, (Tese de doutorado não publicada), USP, 1981.

BRASIL, Lina S. B. Gamificação aplicada ao módulo autoinstrucional online "Saúde da população negra”. Jornal Brasileiro de Telessaúde, Rio de Janeiro, v. 4, n. 2, p. 299-301, 2016. Disponível em: https://www.epublicacoes.uerj.br/index.php/jbtelessaude/article/view/33583 Acesso em 31 jul. 2020

BRUHN, Allison L.; BALINT-LANGEL, Kinga; TROUGHTON, Leonard; LANGAN, Sean; LODGE, Kelsey; KORTEMEYER, Sara. Assessing and treating stereotypical behaviors in classrooms using a functional approach. Behavioral Disorders, v. 41 n. 1 p. 21-37, 2015. Disponível em https://doi.org/10.17988/0198-7429-41.1.21 Acesso em 15 mar. 2021

CARVALHO NETO, Marcus Bentes. Análise do Comportamento: Behaviorismo radical, análise experimental do comportamento e análise aplicada do comportamento. Interação em Psicologia, v. 6, n. 1, p 13-18, 2002. Disponível em http://dx.doi.org/10.5380/psi.v6i1.3188 Acesso em 29 mar 2021.

CONSTANCIO, Fernando G.; NOGUEIRA, Danielle X. P.; COSTA, João P. C. L. Proposta de modelo ADDIE estendido com aplicação nos cursos autoinstrucionais mediados por tecnologias na escola nacional de administração pública. Anais do Encontro Virtual de Documentação em Software Livre e Congresso Internacional de Linguagem e Tecnologia Online, Minas Gerais, 2016. Disponível em: http://www.periodicos.letras.ufmg.br/index.php/anais_linguagem_tecnologia/article/view/105 06_Acesso em 31 jul. 2020 
http://dx.doi.org/10.5902/1984686X54536

DE BONA, Michele. Análise do treinamento autoinstrucional no ambiente de agência bancária: estudo de caso na agência T. (Trabalho de conclusão de curso não publicado), Rio Grande do Sul, 2010. Disponível em: https://ume.ufrgs.br/handle/10183/29813 Acesso em 31 jul. 2020

DIGGENARO, Florence D.; MARTENS Brian K. A Comparison of Performance Feedback Procedures on Teachers' Treatment Implementation Integrity and Students' Inappropriate Behavior in Special Education Classrooms. Journal of applied behavior analysis, v. 40, n. 3, p. 447-461. Disponível em: 10.1901/jaba.2007.40-447 Acesso em 29 mar. 2021.

FAÉ, Rogério. Aprendizagem organizacional: um estudo de caso sobre Ensino à Distância. Revista da FAE, v. 15, n. 2, p. 68-83, 2012. Disponível em:

https://revistafae.fae.edu/revistafae/article/view/196 Acesso em 31 jul. 2020

FORNAZARI, Sílvia. A. Comportamentos inadequados e produtividade em pessoas com deficiência mental ou múltipla em ambiente educacional. (Tese de doutorado não publicada), UNESP, Araraquara, 2005.

FRITZ, Jennifer. N.; IWATA, Brian. A.; ROLIDER, Natalie. U.; CAMP, Erin. M.; NEIDERT, Pamela. L. Analysis of self-recording in self-management interventions for stereotypy. Journal of Applied Behavior Analysis, v. 45, n. 1, p. 55-68, 2012. Disponível em: https://www.ncbi.nlm.nih.gov/pmc/articles/PMC3297353/ Acesso em 31 jul. 2020

\section{HAMADA, Raquel A.; FORNAZARI, Sílvia A. Material didático de capacitação para pais em Análise do comportamento (Material não publicado), 2014}

HAMADA, Raquel. A. Comportamentos aberrantes: revisão de estudos sobre tema e elaboração de material didático para intervenção com pais. (Dissertação não publicada), UEL, Londrina, 2014. Disponível em: http://www.uel.br/pos/pgac/wpcontent/uploads/2014/03/Comportamentos-aberrantes-revisão-de-estudos-sobre-o-tema-eelaboração-de-material-didático-para-intervenção-com-pais.pdf Acesso em 31 jul. 2020

HEITZMAN-POWELL, Linda S.; BUZHARDT, Jay; RUSINKO, Lisa C.; MILLER, Todd M. Formative evaluation of an ABA outreach training program for parents of children with Autism in remote areas. Focus on Autism and Other Developmental Disabilities, v. 29, n. 1, p. 23-38, 2013. Disponível em:

https://journals.sagepub.com/doi/abs/10.1177/1088357613504992 Acesso em 31 jul. 2020

HENKLAIN, Marcelo H. O.; CARMO, João S. Contribuições da Análise do Comportamento à Educação: um convite ao diálogo. Cadernos de Pesquisa, v. 43, n. 149, p. 704-723, 2013. Disponível em: https://www.scielo.br/pdf/cp/v43n149/16.pdf Acesso em 31 jul. 2020

HIGGINS, William J.; LUCZYNSKI, Kevin C.; CARROL, Regina A.; FISHER, Wayne W.; MUDFORD, Oliver $C$. Evaluation of a telehealth training package to remotely train staff to conduct a preference assessment. Journal of Applied Behavior Analysis, v. 50, n. 2, p. 238-251, 2017. Disponível em: https://pubmed.ncbi.nlm.nih.gov/28090644/ Acesso em 31 jul. 2020

KELLER, Fred. S.; SHERMAN, J. Gilmour. The Keller plan handbook. Menio Park, W.A, Benjamin, 1974. 
KIENEN, Nádia.; KUBO, Olga. M.; BOTOMÉ, Sílvio. P. Ensino programado e programação de condições para o desenvolvimento de comportamentos: alguns aspectos no desenvolvimento de um campo de atuação do psicólogo. Acta Comportamentalia, v. 21, n. 4, p. 481-494, 2013. Disponível em: http://pepsic.bvsalud.org/pdf/actac/v21n4/n4a06.pdf Acesso em 31 jul. 2020

KOEGEL, Lynn; MATOS-FREDEN, Rosy; LANG, Russell; KOEGEL, Robert Interventions for children with autism spectrum disorders in inclusive school settings. Cognitive and Behavioral Practice, v. 19, n. 3, p. 401-412, 2012. Disponível em: https://psycnet.apa.org/record/2011-09078-00 Acesso em 31 jul. 2020

KUBO, Olga. M.; BOTOMÉ, Sílvio. P. Ensino-aprendizagem: uma interação entre dois processos comportamentais. Interação em Psicologia, v. 5, n. 1, p. 9-12, 2001. Disponível em: https://revistas.ufpr.br/psicologia/article/view/3321/2665 Acesso em 31 jul. 2020

LUISELLI, James K.; BASS, Jennifer D.; WHITCOMB, Sara. A. Teaching applied behavior analysis knowledge competencies to direct-care service providers: outcome assessment and social validation of a training program. Behavior Modification, v. 34, n. 5, p. 403-414, 2010. Disponível em: https://pubmed.ncbi.nlm.nih.gov/20935241/ Acesso em 31 jul. 2020

LUNA, Sérgio V. A crise da educação e o Behaviorismo. Que parte nos cabe nela? Temos soluções a oferecer? In K. CARRARA (Org.), Educação, Universidade e Pesquisa. III Simpósio em Filosofia e Ciência: Paradigmas do Conhecimento no Final do Milênio. São Paulo, 2001.

MAGLIONE, Margaret; KADIYALA, Srikanth; KRESS, Amii; HASTINGS, Jaime L.; O'HANLON, Claire E. TRICARE Applied Behavior Analysis (ABA) Benefit: Comparison with Medicaid and Commercial Benefits. Rand Health, v. 13, n. 6, p. 2-10, 2017. Disponível em https://www.ncbi.nlm.nih.gov/pmc/articles/PMC5568164/ Acesso em 29 mar. 2021.

MARIN, Ramon; FALEIROS, Pedro B.; MORAES, Antonio B. A. Como a Análise do Comportamento tem Contribuído para Área da Saúde? Psicologia: ciência e profissão, v. 40, n. 12, p. 1982-3703, 2020. Disponível em: https://doi.org/10.1590/1982-3703003197787 Acesso em 29 mar. 2021.

MATOS, M. A. Análise funcional do comportamento. Revista Estudos em Psicologia, v. 16, n. 3, p. 8-18, 1999. Disponível em:

https://www.scielo.br/pdf/estpsi/v16n3/a02v16n3.pdf Acesso em 31 jul. 2020

MILTERNBERGER, Raymond G. Modificação do comportamento: teoria e prática. Cengage, São Paulo, 2018.

MOORE, James W.; EDWARDS, Ron P.; STERLING, Heather; RILEY, Julie. Teacher acquisition of functional analysis methodology. Journal of applied behavior analysis, v. 35, n. 1, p. 35-73, 2002. Disponível em https://doi.org/10.1901/jaba.2002.35-73.

Acesso em 28 de mar. 2021. 
MUELLER, Michael. M.; PIAZZA, Cathleen. C.; MOORE, James. W.; KELLEY, Michael. E.; BETHKE, Stephanie. A.; PRUETT, Angel. E.; OBERDORFF, Amanda. J.; LAYER, Stacy. A. Training parents to implement pediatric feeding protocols. Journal of Applied Behavior Analysis, v. 36, n. 4, p. 545-562, 2003. Disponível em:

https://www.ncbi.nlm.nih.gov/pmc/articles/PMC1284468/ Acesso em 31 jul. 2020.

NASCIMENTO, Gabriela A.; SOUZA, Sandra F. A inclusão de alunos com transtorno do espectro autista (TEA): possibilidades de intervenção psicopedagógica através da Análise do comportamento aplicada, Paidéia, v. 13, n. 19, p. 163-185, 2018. Disponível em: http://www.fumec.br/revistas/paideia/article/view/6322/3136 Acesso em 31 jul. 2020

OLIVER, Anthony C.; PRATT, Leigh A.; NORMAND, Matthew P. A survey of functional behavior assessment methods used by behavior analysts in practice. Journal of Applied Behavior Analysis, v. 48, n. 4, p. 817-829, 2015. Disponível em: https://www.researchgate.net/publication/282305958_A_survey_of_functional_behavior_ assessment_methods_used_by_behavior_analysts_in_practice Acesso em $3 \overline{1}$ jul. 2020

OLTHUIS, Janine V.; MCGRATH, Patrick J.; CUNNINGHAM, Charles E.; BOYLE, Michael H.; POTTIE-LINGLEY; Patricia, REID; Graham J. Reid; BAGNELL, Alexa; LIPMAN, Ellen L.; TURNER, Karen; CORKUM, Penny; STEWART, Sherry H.; BERRIGAN, Patrick; SDAO-JARVIE, Kathy. Distance-Delivered Parent Training for Childhood Disruptive Behavior (Strongest Families ${ }^{\mathrm{TM}}$ ): A Randomized Controlled Trial and Economic Analysis. Journal of Abnormal Child Psychology, v. 46, n. 8, p. 16131629, 2018. Disponível em: https://pubmed.ncbi.nlm.nih.gov/29516341/ Acesso em 31 jul. 2020

PINHEIRO, Maria I. S.; HAASE, Vitor G., PRETTE, Almir D., AMARANTE, Claret L. D., PRETTE, Zilda A. P. D. Treinamento de habilidades sociais para pais de crianças com problemas de comportamento. Psicologia: reflexão e crítica, v. 19, n.3, p. 407- 414. 2006. Disponível em: https://doi.org/10.1590/S0102-79722006000300009 Acesso em 28 mar. 2021.

QUERIM, Angie C.; IWATA, Brian A.; ROSCOE, Eileen M.; SCHLICHENMEYER, Kevin J.; ORTEGA, Javier. V.; HURL, Kylee E. Functional analysis screening for problem behavior maintained by automatic reinforcement. Journal of Applied Behavior Analysis, v. 46, n. 1, p. 47-60, 2013. Disponível em: https://www.researchgate.net/publication/257647685_Functional_analysis_screening_for _problem_behavior_maintained_by_automatic_reinforcement Acesso em 31 jul. 2020

REED, Florence D. D.; REED, Derek. D.; BAEZ, Cynthia. N.; MAGUIRE, Helena. Parametric analysis of errors of commission during discrete-trial training. Journal of Applied Behavior Analysis, v. 44, n. 3, p. 611-615, 2011. Disponível em: https://www.ncbi.nlm.nih.gov/pmc/articles/PMC3177342/ Acesso em 31 jul. 2020

SOUZA, Silvia Regina; GONGORA, Maura. Análise do comportamento e a psicologia do esporte: alguns esclarecimentos, Revista Brasileira de Psicologia do Esporte, São Paulo, v. 6, n. 1, 2016. DOI: http://dx.doi.org/10.31501/rbpe.v6i1.6719 
STEINBRENNER, Jessica R.; HUME, Kara; ODOM, Samuel L.; MORIN, Kristi L.; NOWELL, Sallie W.; TOMASZEWSKI, Brianne; SZENDREY, Susan; Mclntyre, Nancy S.; YUCESOY-OZKAN, Serife; SAVAGE, Melissa N. Evidence-based practices for children, youth, and young adults with autism. The University of North Carolina at Chaper Hill, Frank Porter Graham Child Development Institute, National Clearinghouse on Autism evidence and practice review team, 2020. Disponível em https://ncaep.fpg.unc.edu/sites/ncaep.fpg.unc.edu/files/imce/documents/EBP\%20Report\% 202020.pdf Acesso em 15 mar de 2021.

STUMPHAUZER, Jerome S.; DAVIS, Lynda C. Training Mexican American mental health in personnel in behavior therapy. Journal of Therapy and Experimental Psychiatry, v. 14, n. 3, p. 215-217, 1983. Disponível em: https://www.sciencedirect.com/science/article/abs/pii/0005791683900514 Acesso em 31 jul. 2020

TOURINHO, Emmanuel Z. Estudos conceituais na análise do comportamento. Temas em Psicologia da SBP, São Paulo, v. 7, n. 3, p. 213-222, 1999. Disponível em: http://pepsic.bvsalud.org/scielo.php?script=sci_arttext\&pid=S1413-389X1999000300003 Acesso em 29 mar 2021.

VOLLMER, Timothy; IWATA, Brian A. Differential reinforcement as treatment for behavior disorders: procedural and functional variations. Research in Developmental Disabilities, v. 13, n. 4, p. 393-417, 1993. 394 p. Disponível em: 10.1016/0891-4222(92)90013-V Acesso em 31 jul. 2020

YAZAWA, Thaís. Comportamentos aberrantes: revisão de procedimentos de intervenção, elaboração e avaliação de material didático para profissionais da saúde. (Dissertação não publicada) Universidade Estadual de Londrina, UEL, 2015. Disponível em: http://www.uel.br/pos/pgac/wp-content/uploads/2016/02/Comportamentosaberrantes-revisão-de-procedimentos-de-intervenção-elaboração-e-avaliação-de-materialdidático-para-profissionais-da-saúde.pdf Acesso em 31 jul. 2020

\section{YAZAWA, Thaís; FORNAZARI, Sílvia A. Graves problemas de comportamento no} atendimento em saúde: como lidar? (Material de capacitação não publicado), 2015.

\section{YAZAWA, Thaís; FORNAZARI, Sílvia. A.; RODRIGUES, Olga M. P. R. R. Graves} problemas de comportamento no atendimento em saúde: como lidar? (Material de capacitação não publicado), 2018.

YOSHIY, Shimeny M.; KIENEN, Nádia Elaboração de um livro autoinstrucional para desenvolver "gerenciamento de tempo" em estudantes universitários. In KIENEN, N.; GIL, S. R. S. A., LUZIA, J. C.; GAMBA, J. (org). Análise do comportamento: conceitos e aplicações a processos educativos, clínicos e organizacionais. Londrina, UEL, 2018. p. 66-84. Disponível em:

https://www.researchgate.net/profile/Paulo_Do_Prado/publication/330479753_UELlivro5d ez18press/links/5c41ce8a299bf12be3d17b85/UELlivro5dez18press.pdf. Acesso em 31 jul. 2020. 